\title{
Correction to: The spatial distribution of Republican and Democratic climate opinions at state and local scales
}

\author{
Matto Mildenberger ${ }^{1}$ • Jennifer R. Marlon ${ }^{2}$ - Peter D. Howe ${ }^{3} \cdot$ Anthony Leiserowitz $^{2}$
}

Published online: 29 December 2017

(C) Springer Science+Business Media B.V., part of Springer Nature 2017

\section{Correction to: Climatic Change (2017) 145:539-548 \\ https://doi.org/10.1007/s10584-017-2103-0}

The referenced publication included a methodological error that affects a portion of the reported results for registered Democrats by about 1 percentage point on average.

The original publication provides estimates of the distribution of public opinion among Republicans and Democrats. The estimates were constructed by combining survey data with US voter file data, census American Community Survey (ACS) data, and other data in a multilevel regression model. The voter file data are cross-tabulated counts of containing the number of American adults in different partisan and demographic subgroups and for each unique geographic area (i.e., states and congressional districts).

Estimates for registered Democrats were in error because we mislabeled a subset of Democratic district-level voter file crosstabs at an early stage in the data preparation process. As a result, the subpopulation numbers for the Democratic opinion estimates were based on the number of Republicans in each demographic-geographic subtype

The online version of the original article can be found at https://doi.org/10.1007/s10584-017-2103-0

Matto Mildenberger

mildenberger@polsci.ucsb.edu

1 Department of Political Science, University of California Santa Barbara, Santa Barbara, CA 93106-9420, USA

2 School of Forestry and Environmental Studies, Yale University, 195 Prospect Street, New Haven, CT 06511, USA

3 Department of Environment and Society, Quinney College of Natural Resources, Utah State University, 5215 Old Main Hill, Logan, UT 84322-5215, USA 
rather than on the number of Democrats, in the subset of 32 states with party registration data.

The error does not affect the Republican opinion estimates, and has a minimal effect on the Democratic opinion estimates. Most estimates of Democratic opinion at state and congressional district levels change no more than 1 percentage point. On average, the corrected estimates are 1.2 percentage points lower at the state level and 1.3 percentage points lower at the district level than the previous estimates. The corrected estimates are highly correlated with the previous estimates (mean $r=0.97$ ). The new values are also all within the margin of error of the previous values, with the sole exception of North Carolina's 12 District where the revised Democratic estimates fall slightly outside the previous values for two opinions (belief global warming is human-caused and belief there is a scientific consensus around global warming).

Figures 2, 3, and 4 here replace Figs. 2, 3, and 4, respectively, in the paper.

In the sixth paragraph of section 2 Results (beginning on page 543 of the printed journal), the reported variation among Democratic partisans changes somewhat relative

a

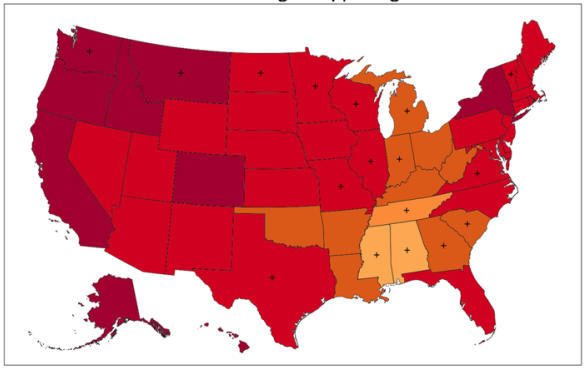

Projected \% of registered Democrats, 2016 \begin{tabular}{llllllllllllllllllllll}
\hline 0 & 5 & 10 & 15 & 20 & 25 & 30 & 35 & 40 & 45 & 50 & 55 & 60 & 65 & 70 & 75 & 80 & 85 & 90 & 95
\end{tabular}

C



Projected \% of registered Democrats, 2015 $\begin{array}{lllllllllllllllll}0 & 0\end{array}$ b

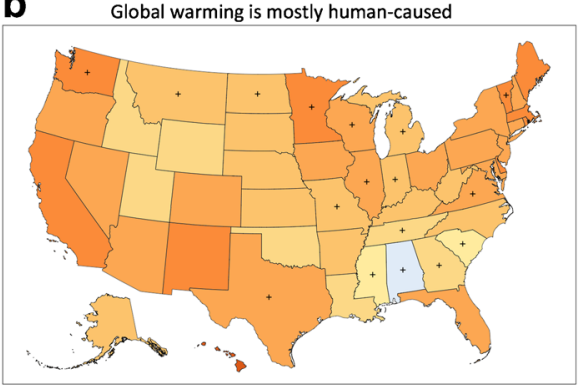

Projected \% of registered Democrats, 2016

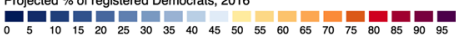

d Support regulation of $\mathrm{CO} 2$ as a pollutant

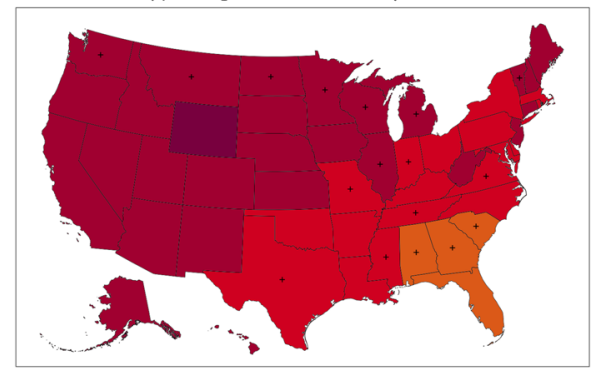

Projected \% of registered Democrats, 2016

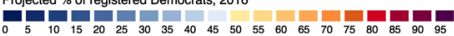

Fig. 2 Projected \% of Democrats who hold selected climate opinions and policy preferences by state. States with plus symbol do not provide party registration data; party identification in these states is modeled 

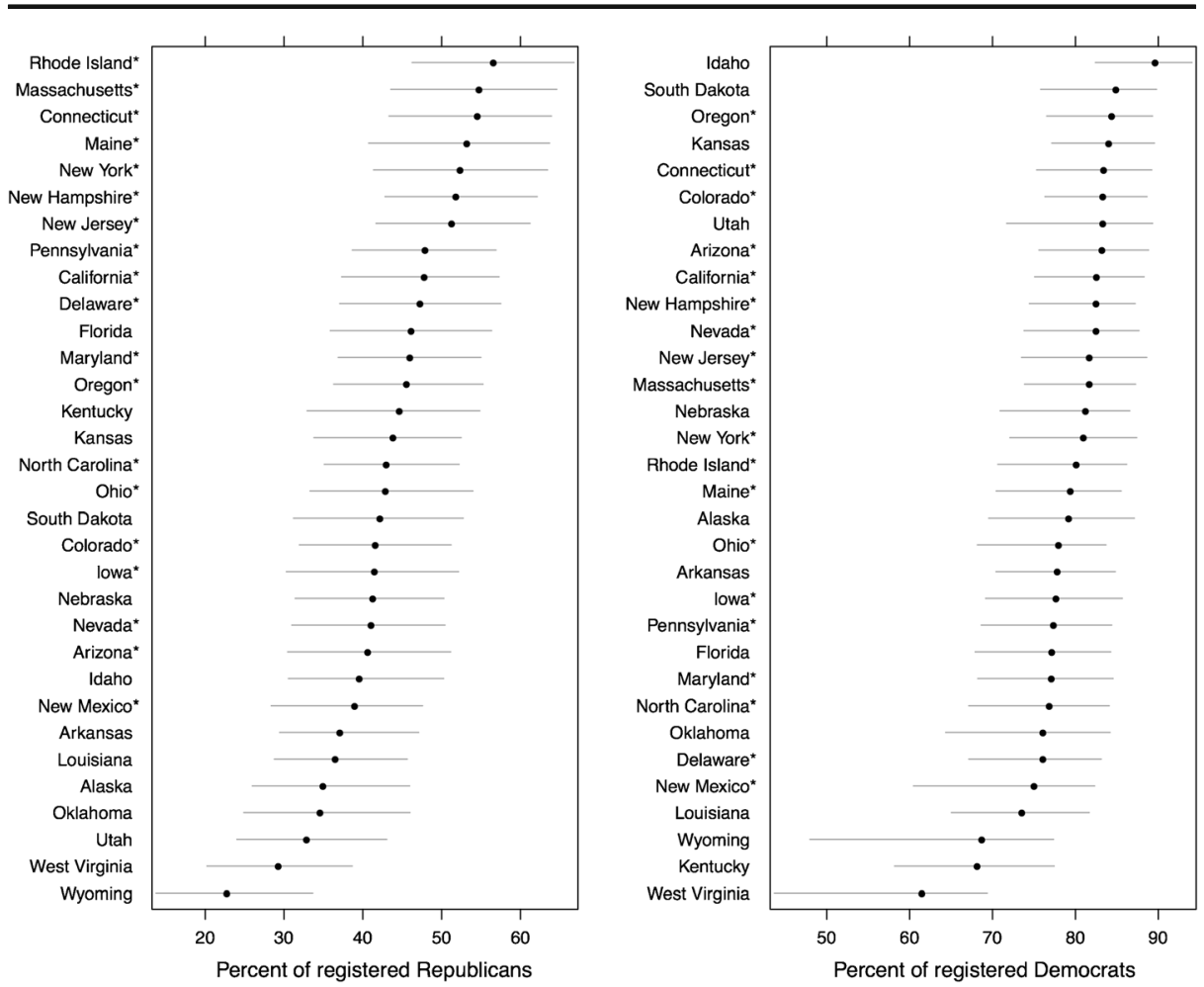

Fig. 3 Projected \% of registered Republicans (left) and Democrats (right) supporting state-level Renewable Portfolio Standards by state (states with party registration ID only). Asterisk indicates states with current Renewable or Alternate Energy Portfolio Standards

to Republicans, but substantive conclusions remain similar. This paragraph should now read:

Importantly, we find heterogeneity in congressional-district level opinions among both Democrats and Republicans. For belief in climate change, the standard deviation of district-level opinions is similar among both Democrats and Republicans. For this item, the primary difference is that the Democratic spread is centered around a much higher partisan average. However, for some policy items, we see evidence of substantially less heterogeneity among Democrats as compared to Republicans. For instance, the standard deviation across congressional districts in support for carbon pollution regulation is only 3.2 percentage points among Democratic partisans but substantially higher (5.4 percentage points) among Republicans. By contrast, for the worry about climate change item, we find the opposite result with Democratic heterogeneity (6.7 percentage points) being somewhat higher than Republicans (4.7 percentage point). However, despite 


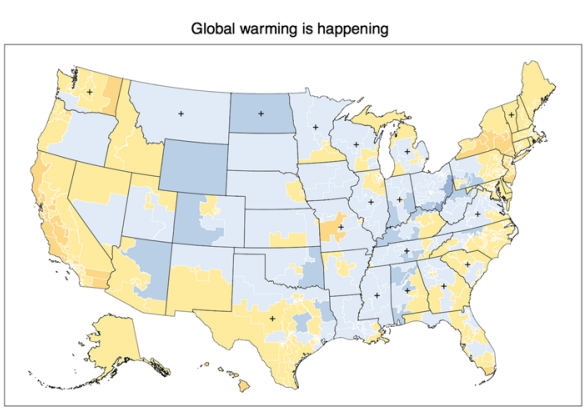

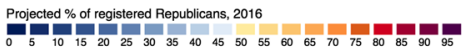

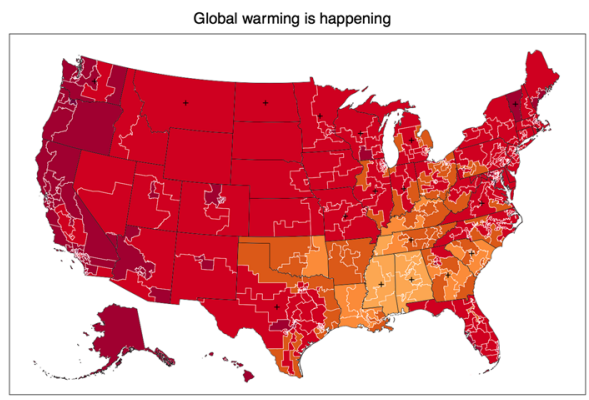

Projected \% of registered Democrats, 2016

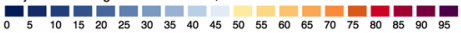

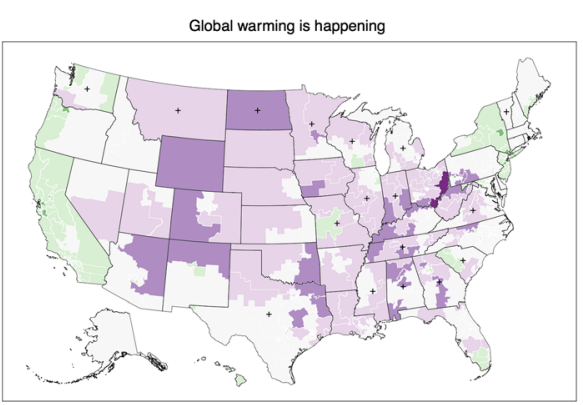

Difference from US Republican average, 2016 (53\%) $\begin{array}{lllllll}-1450-11 \% & -1010-7 \% & -60-3 \% & -210 \% & 2105 \% & 009 \% & 101010 \%\end{array}$

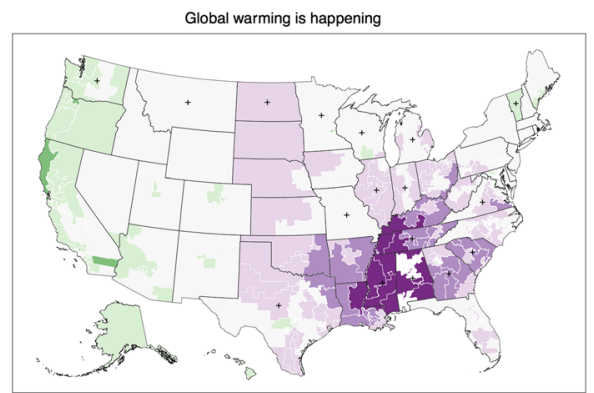

Difference from US Democratic average, 2016 (82\%)

$-1710-11 \% 6-1010-7 \% \quad-610-9 \% \quad-201 \% \quad 2105 \% \quad 610 \% \quad 10 \% 14 \%$

Fig. 4 Projected \% of registered Republicans (top row) and Democrats (bottom row) who believe global warming is happening by congressional district. Left panels map absolute estimates and right panels map relative differences from the national average for Republicans and Democrats, respectively. Congressional districts in states with plus symbol do not provide party registration data; party identification in these districts is modeled

variation in Democratic intensity of support, even Democrats in the lower tail of the congressional district distribution still hold absolute levels of support compatible with policy reform.

All data and the interactive visualization tool have been updated to reflect the corrected values. 